\title{
Categorization of Chinese Dry-Cured Ham Based on Three Sticks' Method by Multiple Sensory Technologies
}

\author{
Dong Xia, ${ }^{1}$ Dan Ni Zhang, ${ }^{1,2}$ Shao Ting Gao, ${ }^{1}$ Le Chen, ${ }^{1}$ Nan Li, \\ Fu Ping Zheng, ${ }^{3,4}$ and Yuan Liu ${ }^{1,2,3,4}$ \\ ${ }^{1}$ College of Food Science and Technology, Shanghai Ocean University, Shanghai 201306, China \\ ${ }^{2}$ Department of Food Science and Technology, School of Agriculture and Biology, Shanghai Jiao Tong University, \\ Shanghai 200240, China \\ ${ }^{3}$ Beijing Advanced Innovation Center for Food Nutrition and Human Health, Beijing Technology \& Business University, \\ Beijing 100048, China \\ ${ }^{4}$ Beijing Laboratory for Food Quality and Safety, Beijing Technology and Business University, Beijing 100048, China
}

Correspondence should be addressed to Fu Ping Zheng; zhengfp@th.btbu.edu.cn and Yuan Liu; y_liu@sjtu.edu.cn

Received 21 July 2017; Accepted 11 September 2017; Published 18 October 2017

Academic Editor: Jesús Lozano

Copyright (C) 2017 Dong Xia et al. This is an open access article distributed under the Creative Commons Attribution License, which permits unrestricted use, distribution, and reproduction in any medium, provided the original work is properly cited.

\begin{abstract}
Odor profiles of three grades of Jinhua, Xuanwei, and Rugao dry-cured hams were analyzed and distinguished by both the electronic nose and the sensory evaluation. The odor was absorbed by bamboo sticks, which is the most traditional absorption method to classify different ham grades. Then data from electronic nose was analyzed by discriminant function analysis (DFA) and cluster analysis (CA), compared with that from sensory evaluation by principal component analysis (PCA). Results showed that different grades of Jinhua, Xuanwei, and Rugao dry-cured hams could be distinguished effectively by the DFA results of electronic nose. However, sensory evaluation could not perform as well as electronic nose. It was demonstrated that intelligent sensory technology has higher sensitivity and reliability in classifying producing regions and grades of dry-cured ham.
\end{abstract}

\section{Introduction}

Dry-cured hams are the traditional and popular meat products in China, because of their unique flavor and long-term preservation. Jinhua, Rugao, and Xuanwei dry-cured hams are three of the most well-known ham products in China. Jinhua hams are produced in Jinhua, Zhejiang Province, made from the hind legs of pigs [1] of Jinhua two-end black. Xuanwei hams were produced in Xuanwei, Yunnan Province, which are an example of the earliest famous foods of Yunnan to enter the international market. They are made from hind legs [2] of Wujin pig in Yunnan. Because of the high quality of muscles, Wujin pigs are ideal raw materials for producing the high-quality Xuanwei hams [3]. Rugao hams are called "the North Leg," whose history can be traced back to the Qing Dynasty. They are produced in Rugao, Jiangsu Province, made from the local Rugao pigs [4]. The high quality of the dry-cured ham mainly accompanies the delightful aroma, which is one of the most important labels of dry-cured hams.

The traditional way of grading the dry-cured ham is called "Three Sticks' Method" which depends on ham technicians with extensive experiences to smell the aroma adsorbed on the bamboo sticks. The bamboo sticks are stuck in the sacral vertebra, knee joint, and hip joint of hams. But the aroma from the hip joint is the most difficult to form, so the odor from that position can speculate the quality of the ham. Although the traditional method has a long history, it still has many disadvantages. For example, it has strong subjectivity and weak repeatability which may vary by different factors, such as the physiological and mental reasons or the experiences of ham technicians. So the traditional method can no longer adapt to the modern requirements. With the development of modern technology, intelligent sensory technology such as electronic nose was invented to 
make up for these deficiencies. Electronic nose has been successfully used to identify the different qualities of crab [5], olive oil [6], tea [7], sheep [8], and other products. The electronic nose is an intelligent system which can imitate the human's senses of olfaction and distinguish different samples by pattern recognition systems. It has been applied widely in food science, environmental monitoring, and other fields because of its simple operation, high sensitivity, and fast response $[9,10]$. There have been a number of scholars using it to identify and analyze the quality of dry-cured ham [11-13]. Santos et al. [14] used the electronic nose to identify Iberian hams with different feeding conditions and ripening time. But there has been no research on the electronic nose (Three Sticks' Method) to identify the grades and producing areas of dry-cured hams instead of sensory evaluation.

In this project, Three Sticks' Method was applied to absorb odors of hams as traditional ways. The electronic nose and sensory evaluation were used to classify the different producing regions and grades of Jinhua, Rugao, and Xuanwei dry-cured hams. The aim of this study was to seek an efficient new way to distinguish different categories and grades of drycured hams.

\section{Materials and Methods}

2.1. Materials. The Jinhua hams were produced by Jinzi Corporation Limited Company of Zhejiang Province. The Xuanwei hams were produced by Xuantai Ham Company of Yunnan Province. The Rugao hams were produced by Changshou Ham Limited Company of Jiangsu Province. Every variety has three grades, respectively, which were differentiated by "Three Sticks' Method" according to their producing time and technology (Grades of Ham Samples and Their Abbreviations). Jinhua top-grade ham (JHT), Xuanwei three-year ham (XW3), and Rugao three-year ham (RG3) were considered as the top-grade products. Jinhua first-grade ham (JH1), Xuanwei two-year ham (XW2), and Rugao twoyear ham (RG2) were the second-grade products. Jinhua second-grade ham (JH2), Xuanwei one-year ham (XW1), and Rugao one-year ham (RG1) were the third-grade products. A total of 45 dry-cured hams were analyzed; each grade had 5 samples. All of them were vacuum-packed in the same shape and stored at $-20^{\circ} \mathrm{C}$ after purchase.

2.2. Sample Preparation. Samples were thawed overnight at $4^{\circ} \mathrm{C}$. The visible fat layer and cortex on the surface were removed on sterile props. Bamboo sticks used for "Three Sticks' Method" were put in a cool place to dry for 30 minutes after being soaked in ethanol.

2.3. Electronic Nose Analysis. The electronic nose (FOX $\alpha 4000$, Alpha MOS, France) is composed a 96-position autosampler and an array of gas sensors. The sensor array consists of three metal oxide sensor chambers and 18 sensors. The bamboo sticks were stuck into the hip joint of each sample for 20 mins (Figure 1). They were further transferred to $10 \mathrm{ml}$ glass vials with screw cap. The temperature of the samples was conditioned to refrigerator's temperature at $4^{\circ} \mathrm{C}$, before they were placed in the autosampler of the electronic nose.

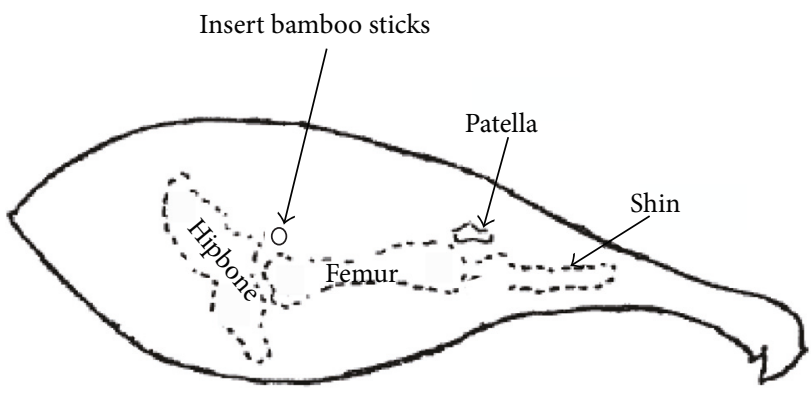

FIgURE 1: Information of insert of the bamboo sticks.

Afterwards, the vials were incubated for $400 \mathrm{~s}$ at $50^{\circ} \mathrm{C}$, after which the aromas in headspace were taken into the sensor array chamber by the sampling pump at a flux speed of $150 \mathrm{~mL} / \mathrm{min}$ for $600 \mathrm{~s}$. Thereafter the sensors were purged by clean dry air at a flux speed of $500 \mu \mathrm{L} / \mathrm{s}$ for $120 \mathrm{~s}$. The maximum response peaks of 18 sensors were analyzed with Alpha Soft software. Each sample was analyzed in octuplicate.

2.4. Sensory Evaluation. In the sensory assessment, quantitative descriptive analysis method and triangle test [15] method were carried out by a group of 15 selected trained assessors for three times (consisting of 8 females and 7 males, aged 20 to 30 years). Each assessor was served nine samples which were prepared like the electronic nose analysis and each sample was given a three-digit number in a randomized design. The samples were evaluated on a scale from 1 to $9(9$, very strong; 7, strong; 5 , moderate; 3 , weak; 1 , very weak) for the following attributes: meaty odor, souse odor, sour odor, oily odor, and refreshing odor after sniffing. At the same time, 18 groups of samples were provided to each assessor for the triangle test. Each group had three samples, while two of them were the same. Assessors need to distinguish the third nonhomologous samples.

2.5. Statistical Analysis. The sensory data and cluster analysis (CA) were initially assessed by SPSS 12.0 statistical package. The principal component analysis (PCA) for sensory evaluation was performed by Origin Pro 9.1. The discriminant function analysis (DFA) of electronic nose was carried out with Alpha Soft software.

\section{Results and Discussion}

3.1. Electronic Nose Analysis. Different grades of three kinds of Chinese dry-cured hams (Jinhua, Xuanwei, and Rugao ham) were analyzed using discriminant function analysis (DFA) by the electronic nose (Figure 2). The contribution of the accumulative variance of the first two discriminant functions (DF1 and DF2) reached 99.763\%. Discriminant function 1 accounted for $81.368 \%$ while discriminant function 2 accounted for $18.395 \%$. It was obvious that the plot could be separated into two different sections. The points corresponding to three grades of Xuanwei ham were in the second and third quadrant of the plot while all the Jinhua and Rugao ham 


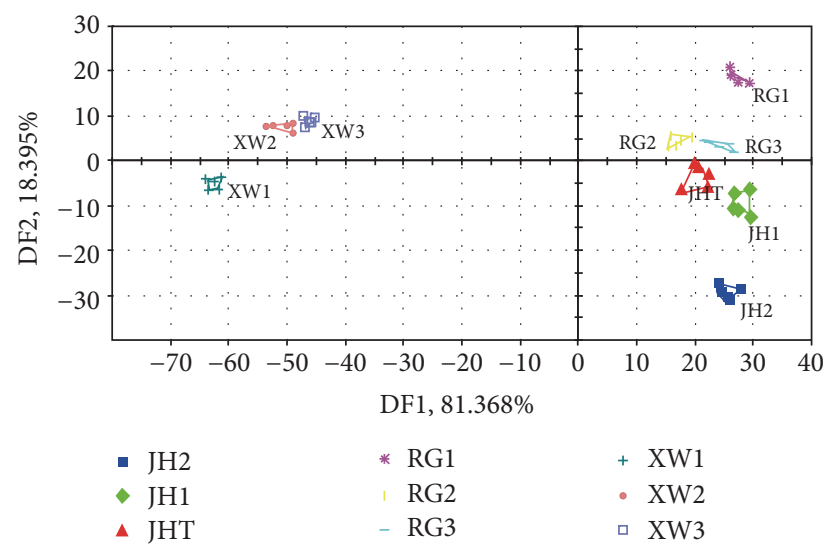

FIGURE 2: Discriminant function analysis (DFA) chart of different grades of three kinds of dry-cured hams by electronic nose. The abbreviations in the figure are referred to in Grades of Ham Samples and Their Abbreviations.

were in the first and fourth quadrant, which suggested that the electronic nose could effectively distinguish the dry-cured ham from different producing areas.

The odor profiles of three grades of Xuanwei hams had huge difference from other two kinds of hams, perhaps because the climate in different regions has a certain impact on the final flavor of the product. Xuanwei hams were produced in Yunnan Province. Yunnan Province is located on the low-latitude plateau of the Northern Hemisphere, where the temperature all year is higher and changes less. In contrast, Zhejiang Province (Jinhua hams) and Jiangsu Province (Rugao hams) are in the east of China, where temperature is higher and has more differences between summer and winter. The particular climate causes the moisture content of Xuanwei hams to be much higher than Jinhua and Rugao hams, and the lipid content is lower, probably leading to the unique flavor of Xuanwei hams [16]. It is well known that lipid is an important source of flavor substances [17]. There is some evidence of a positive effect of temperature on lipolysis phenomena. Andres found that the amount of FFA increased remarkably during the stages of processing with the temperature rising [18]. In addition, aldehydes, carboxylic acids, and alcohols were major volatile compounds in Jinhua [19] and Rugao [4] hams, while the aldehydes, hydrocarbons, and alcohols played an important role in aromas of Xuanwei ham [20]. The contents and compositions of the aroma substances may also cause the difference among the overall odor characteristics of hams [21].

A hierarchical cluster analysis (CA) was applied to classify 45 dry-cured ham samples based on the response signals of 18 sensors (Figure 3). It was revealed that the dry-cured ham samples were divided into two groups at a distance of 13. The first group included all Xuanwei ham samples, while all Jinhua and Rugao ham samples formed the second group. It indicated that Xuanwei ham had a unique odor, which had large differences among Jinhua and Rugao ham. Also, CA gave us a preliminary classification where odor profiles of JH2, XW1, and RG1 had large differences from other two grades of samples of the same origin, respectively. It is probably because the moisture content of one-year hams was still at a high level [22], and some important aroma compounds such as 2-methylbutyraldehyde, 1-octen3-ol, octanal, nonanal, hexanal, heptanal, benzaldehyde, and phenyl acetaldehyde had not been generated completely [23]. Meanwhile, the odor profiles of JH1 and JHT, XW2 and XW3, and RG2 and RG3 were much closer, which was similar to the previous study $[24,25]$.

3.2. Sensory Evaluation. According to the results of DFA and CA, the odor profiles of dry-cured ham were diverse by different regions and grades. In order to understand the relationship between intelligent sensory analysis and human sensory analysis, the principal component analysis (PCA) for sensory evaluation was conducted with the data from the scales of 15 panelists.

The PCA result of dry-cured hams by sensory evaluation was shown in Figure $4.87 .47 \%$ of the variability was explained by the first two principal components. Principal component 1 is the most important variable as it explains $75.13 \%$ of the variability. On the whole, the plot can be divided into two parts, which is consistent with the DFA and CA chart. The odors of Xuanwei ham were distinct obviously. The souse aroma and refreshing aroma of Xuanwei hams were more intense than other two types of hams, while the meaty aroma was the weakest. It is reported that the content of sulphur aroma compounds in Xuanwei hams was less than Jinhua and Rugao hams [16]. Sulphur aroma compounds formed from sulphur containing amino acids, such as methionine, cysteine, and cysteine via Strecker degradations, and they are important contributors to meaty flavor due to their low odor threshold [26]. This might be one of the reasons why Xuanwei ham has a weak meaty odor and owns a special flavor. In addition, the sour aroma of JH2 and RG1 was stronger. This is in agreement with previous work. Ruiz et al. reported that the amount of 2-methylbutanal and 3-methylbutanal increased as processing time went by. Higher scores of 2-methylbutanal and 3-methylbutanal indicated better flavor of hams and lower scores mean rancidity [27]. In this way, dry-cured hams with a long processing time should have a stronger meaty odor. However, in our study the meaty odor of RG2 and JH1 was more intense than RG3 and JHT. This might be because the meaty odor was covered by cured and oily odor with ripening drying time extending [28]. This may explain the oily odor of RG3 and JHT was significantly stronger than others as well as the meaty odor of RG3 and JHT was weaker than RG2 and JH1. Furthermore, the flavor profiles of XW2 and XW3 were much more similar. The results were in good agreement with the cluster analysis by electronic nose.

The triangle test is mainly used to test the differences between two samples [15]. Table 1 revealed the results to distinguish different grades of hams. JH2, XW1, and RG1 had significant differences from other two grades samples $(P \leq 0.05)$, respectively, which was basically consistent with the results of the electronic nose. Results of Table 2 demonstrated that, except for XW1 and RG1, three grades of Xuanwei hams had extremely significant levels $(P \leq 0.05)$ 


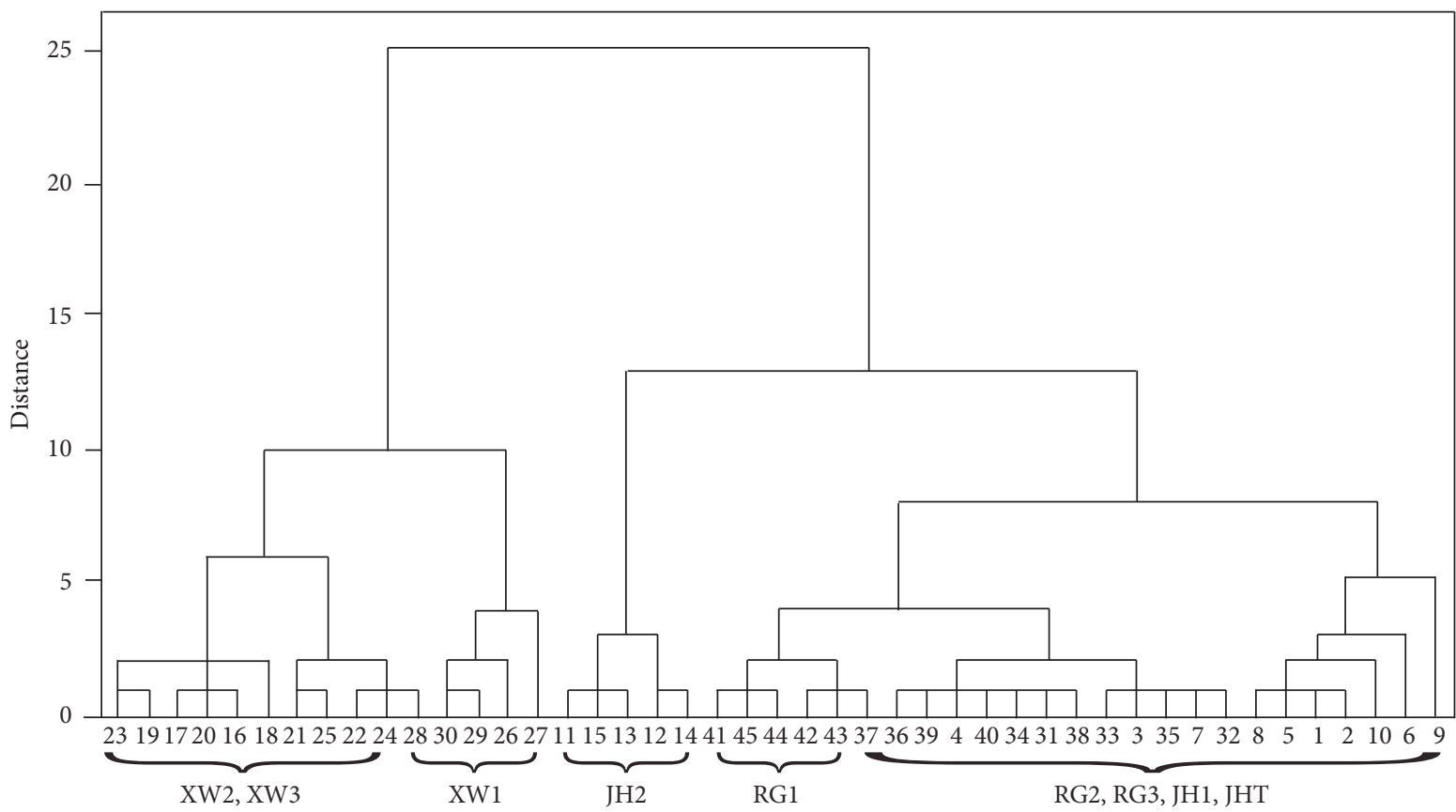

FIGURE 3: Cluster analysis (CA) chart of three kinds of dry-cured hams by the electronic nose. JHT: 1-5, JH1: 6-10, JH2: 11-15, XW3: 16-20, XW2: 21-25, XW1: 26-30, RG3: 31-35, RG2: 36-40, and RG1: 41-45. The abbreviations in the figure are referred to in Grades of Ham Samples and Their Abbreviations.

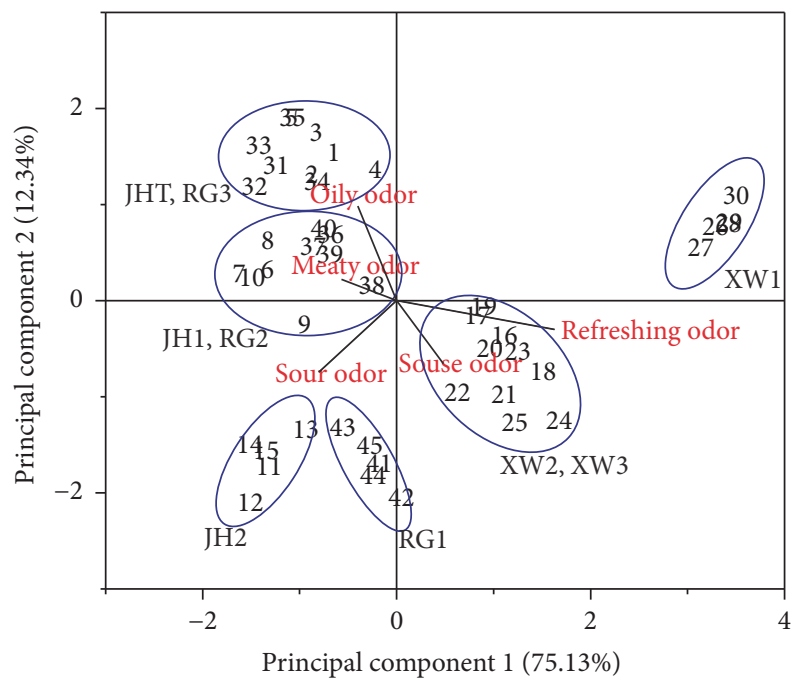

FIGURE 4: Principal component analysis biplot for ham samples and sensory descriptors. JHT: 1-5, JH1: 6-10, JH2: 11-15, XW3: 1620, XW2: 21-25, XW1: 26-30, RG3: 31-35, RG2: 36-40, and RG1: 4145. The abbreviations in the figure are referred to in Grades of Ham Samples and Their Abbreviations.

compared with the same grade of other two brands of hams. In addition, only JHT and RG3 had significant differences by the triangular test, which presented that the flavor profiles of Jinhua and Rugao hams were very similar. The results revealed that the electronic nose nearly could play a better role in categorization of the production regions and grades
TABLE 1: Result of triangle test to distinguish different grades of drycured ham.

\begin{tabular}{lcccc}
\hline Sample & $\begin{array}{c}\text { Number of } \\
\text { correct } \\
\text { choices }\end{array}$ & $\begin{array}{c}\text { Triangle test } \\
\text { inspection } \\
\text { checklist } \\
(n=15) \\
5 \% \quad 1 \%\end{array}$ & $P$ value \\
\hline RG2 \& RG3 & 7 & & & $P>0.05$ \\
RG1 \& RG3 & 9 & & & $P \leq 0.05$ \\
RG1 \& RG2 & 9 & & & $P \leq 0.05$ \\
XW2 \& XW3 & 3 & & & $P>0.05$ \\
XW1 \& XW2 & 9 & 9 & 10 & $P \leq 0.05$ \\
XW1 \& XW3 & 9 & & & $P \leq 0.05$ \\
JH2 \& JH1 & 12 & & & $P \leq 0.01$ \\
JH2 \& JHT & 9 & & & $P \leq 0.05$ \\
JHT \& JH1 & 8 & & $P>0.05$ \\
\hline
\end{tabular}

The abbreviations in the table are referred to in Grades of Ham Samples and Their Abbreviations.

of traditional Chinese dry-cured ham as the human sensory evaluation.

In order to get a better way to compare the identification effect of electronic nose and sensory evaluation, the sensory panel data was analyzed by the cluster analysis (Figure 5). The dry-cured ham samples were separated into three groups by cluster analysis. XW1 had a unique odor, so it formed the first group by itself. RG3, RG2, JHT, JH1, and JH2 are made up of the second group of the CA, which was similar to their 


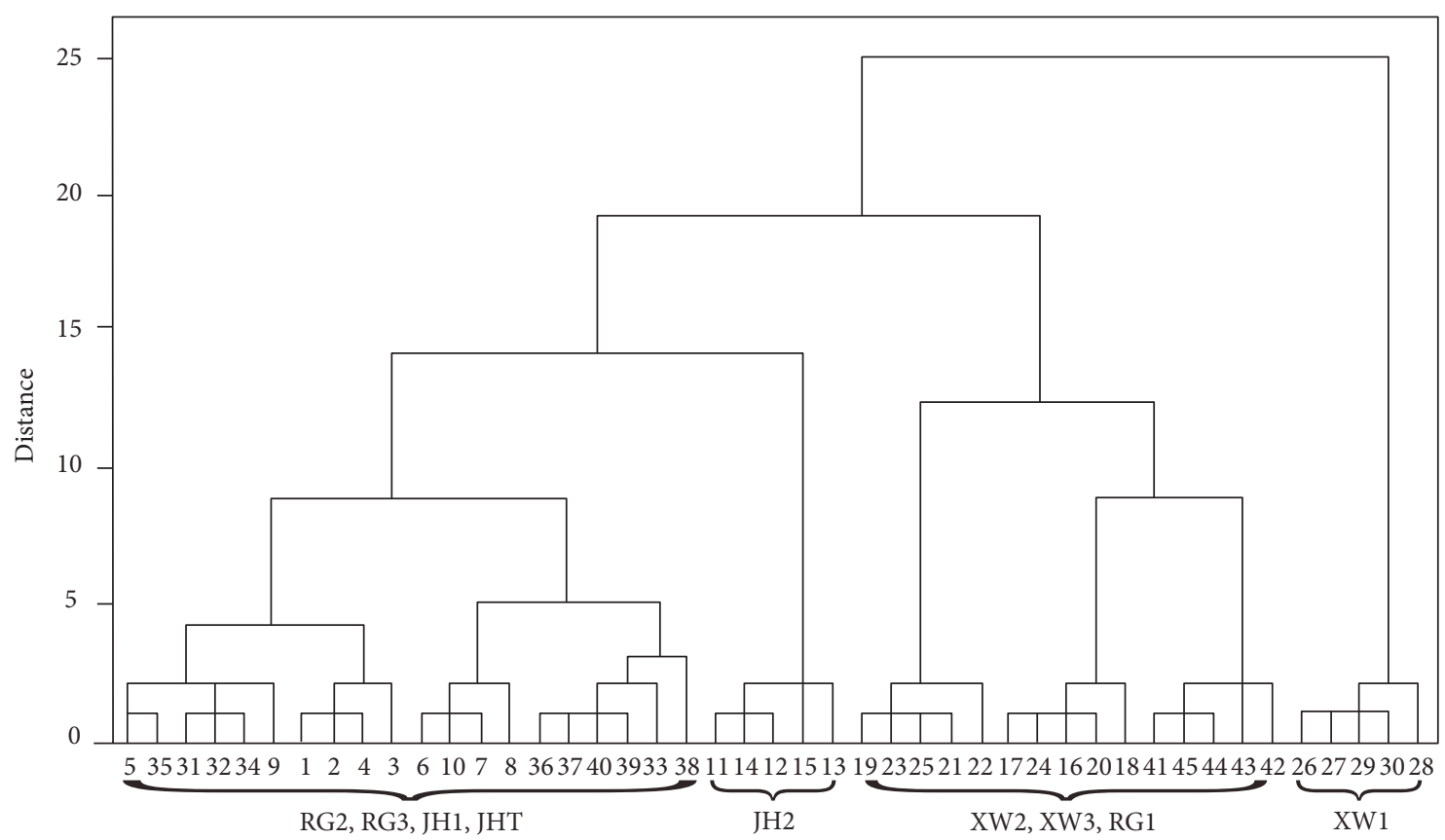

FIGURE 5: Cluster analysis (CA) chart of three kinds of dry-cured hams by sensory evaluation. JHT: 1-5, JH1: 6-10, JH2: 11-15, XW3: 16-20, XW2: 21-25, XW1: 26-30, RG3: 31-35, RG2: 36-40, and RG1: 41-45. The abbreviations in the figure are referred to in Grades of Ham Samples and Their Abbreviations.

TABLE 2: Result of triangle test to distinguish different producing areas of dry-cured ham.

\begin{tabular}{|c|c|c|c|c|c|}
\hline \multirow[t]{2}{*}{ Sample } & \multirow{2}{*}{$\begin{array}{l}\text { Number of } \\
\text { correct } \\
\text { choices }\end{array}$} & \multicolumn{3}{|c|}{$\begin{array}{c}\text { Triangle test } \\
\text { inspection checklist } \\
(n=15)\end{array}$} & \multirow[t]{2}{*}{$P$ value } \\
\hline & & $5 \%$ & $1 \%$ & $0.1 \%$ & \\
\hline RG1 \& XW1 & 7 & \multirow{9}{*}{9} & \multirow{9}{*}{10} & \multirow{9}{*}{12} & $P>0.05$ \\
\hline RG1 \& JH2 & 6 & & & & $P>0.05$ \\
\hline JH2 \& XW1 & 11 & & & & $P \leq 0.05$ \\
\hline RG2 \& XW2 & 13 & & & & $P \leq 0.01$ \\
\hline RG2 \& JH1 & 6 & & & & $P>0.05$ \\
\hline JH1 \& XW2 & 9 & & & & $P \leq 0.05$ \\
\hline RG3 \& XW3 & 9 & & & & $P \leq 0.05$ \\
\hline RG3 \& JHT & 9 & & & & $P \leq 0.05$ \\
\hline JHT \& XW3 & 9 & & & & $P \leq 0.05$ \\
\hline
\end{tabular}

The abbreviations in the table are referred to in Grades of Ham Samples and Their Abbreviations.

classifications by electronic nose. All the XW3, XW2, and RG1 constituted the third group. Cluster analysis is a process of classifying a set of objects into different clusters. Objects in the same cluster are more similar to each other than to those in other groups. It revealed that the odor profiles of WX3, WX2, and RG1 were much closer. The sensory panel is unable to identify RG1 from XW3 and XW2, which proved that electronic nose has a greater ability in distinguishing the Chinese dry-cured ham from different origins and grades.

\section{Conclusions}

In summary, samples with different grades and producing regions could be distinguished by intelligent sensory technology, while human sensory evaluation could not identify parts of samples among the three grades' products as clearly as the electronic nose. This research demonstrated that the electronic nose could reflect the overall odor information of the samples more objectively and sensitively than the artificial sensory evaluation. The application of the electronic nose with "Three Sticks' Method" provides a new way to distinguish dry-cured hams. It may apply on other types of meat products in the near future.

\section{Grades of Ham Samples and Their Abbreviations}

JH2: Jinhua second-grade ham

JH1: Jinhua first-grade ham

JHT: Jinhua top-grade ham

XW1: Xuanwei one-year ham

XW2: Xuanwei two-year ham

XW3: Xuanwei three-year ham

RG1: Rugao one-year ham

RG2: Rugao two-year ham

RG3: Rugao three-year ham.

\section{Conflicts of Interest}

The authors declare that there are no conflicts of interest regarding the publication of this paper. 


\section{Acknowledgments}

This work was funded by Beijing Advanced Innovation Center for Food Nutrition and Human Health and Beijing Laboratory for Food Quality and Safety, Beijing Technology \& Business University (BTBU), National Natural Science Foundation of China (Grant no. 31540087), and the National Key Research and Development Program of China (2016YFD0400803, 2016YFD0401501).

\section{References}

[1] Standardization Administration of the People's Republic of China, Product of geographical indiction-Jinhua ham GB/T 19088-2008, 2008.

[2] Standardization administration of the people's republic of China, Product of geographical indiction-Xuanwei ham GB/T 18357-2008, 2008.

[3] H. Yang, C. Ma, F. Qiao, Y. Song, and M. Du, "Lipolysis in intramuscular lipids during processing of traditional Xuanwei ham," Meat Science, vol. 71, no. 4, pp. 670-675, 2005.

[4] X. L. Zhang, Research on lipoxygenase and flavor in Rugao ham [Master, thesis], Nanjing Agricultural University, Nanjing, China, 2008.

[5] N. Wu, X.-C. Wang, N.-P. Tao, and Y.-Q. Ni, “Odor profiles of hepatopancreas and gonad of Eriocheir sinensis by sensory analysis, electronic nose, and GC-MS analysis," Fisheries Science, vol. 82, no. 3, pp. 537-547, 2016.

[6] D. Melucci, A. Bendini, F. Tesini et al., "Rapid direct analysis to discriminate geographic origin of extra virgin olive oils by flash gas chromatography electronic nose and chemometrics," Food Chemistry, vol. 204, pp. 263-273, 2016.

[7] R. Dutta, E. L. Hines, J. W. Gardner, K. R. Kashwan, and M. Bhuyan, "Tea quality prediction using a tin oxide-based electronic nose: An artificial intelligence approach," Sensors and Actuators B: Chemical, vol. 94, no. 2, pp. 228-237, 2003.

[8] S. Stojković, V. Grabež, M. Bjelanović et al., "Production process and quality of two different dryćured sheep hams from Western Balkan countries," LWT- Food Science and Technology, vol. 64, no. 2, pp. 1217-1224, 2015.

[9] A. D. Wilson and M. Baietto, "Applications and advances in electronic-nose technologies," Sensors, vol. 9, no. 7, pp. 50995148, 2009.

[10] C. Krantz-Rülcker, M. Stenberg, F. Winquist, and I. Lundström, "Electronic tongues for environmental monitoring based on sensor arrays and pattern recognition: A review," Analytica Chimica Acta, vol. 426, no. 2, pp. 217-226, 2001.

[11] M. García, M. Aleixandre, J. Gutiérrez, and M. C. Horrillo, "Electronic nose for ham discrimination," Sensors and Actuators B: Chemical, vol. 114, no. 1, pp. 418-422, 2006.

[12] M. Laureati, S. Buratti, G. Giovanelli, M. Corazzin, D. P. Lo Fiego, and E. Pagliarini, "Characterization and differentiation of Italian Parma, San Daniele and Toscano dry-cured hams: A multi-disciplinary approach," Meat Science, vol. 96, no. 1, pp. 288-294, 2014.

[13] D. L. García-González, N. Tena, R. Aparicio-Ruiz, and R. Aparicio, "Sensor responses to fat food aroma: A comprehensive study of dry-cured ham typicality," Talanta, vol. 120, pp. 342348, 2014

[14] J. P. Santos, M. García, M. Aleixandre et al., "Electronic nose for the identification of pig feeding and ripening time in Iberian hams," Meat Science, vol. 66, no. 3, pp. 727-732, 2004.
[15] Z. Fang, Evaluation of Food Sense Quality, China agricultural press, Beijing, China, 2010.

[16] S. T. Gao, Flavor and quality grade evaluation of dry cured hams in China by multiple fingerprints technology [Master, thesis], Shanghai Ocean Univeisity, Shanghai, China, 2016.

[17] V. Larrea, I. Pérez-Munuera, I. Hernando, A. Quiles, and M. A. Lluch, "Chemical and structural changes in lipids during the ripening of Teruel dry-cured ham," Food Chemistry, vol. 102, no. 2, pp. 494-503, 2007.

[18] A. I. Andres, R. Cava, D. Martin, J. Ventanas, and J. Ruiz, "Lipolysis in dry-cured ham: Influence of salt content and processing conditions," Food Chemistry, vol. 90, no. 4, pp. 523533, 2005.

[19] M. Du and D. U. Ahn, "Volatile substances of Chinese traditional Jinhua ham and cantonese sausage," Journal of Food Science, vol. 66, no. 6, pp. 827-831, 2001.

[20] F. Qiao and C. Ma, "The formation and change of volatile flavor compounds in the process of Xuanwei ham," Food Research and Development, vol. 27, pp. 24-29, 2006.

[21] L. Lorido, M. Estévez, J. Ventanas, and S. Ventanas, "Comparative study between Serrano and Iberian dry-cured hams in relation to the application of high hydrostatic pressure and temporal sensory perceptions," LWT-Food Science and Technology, vol. 64, no. 2, pp. 1234-1242, 2015.

[22] J. Zhang, L. Wang, Y. Liu, J. Zhu, and G. Zhou, "Changes in the volatile flavour components of Jinhua ham during the traditional ageing process," International Journal of Food Science \& Technology, vol. 41, no. 9, pp. 1033-1039, 2006.

[23] M. Narváez-Rivas, E. Gallardo, and M. León-Camacho, “Chemical changes in volatile aldehydes and ketones from subcutaneous fat during ripening of Iberian dry-cured ham. Prediction of the curing time," Food Research International, vol. 55, pp. 381390, 2014.

[24] X. Song, S. T. Gao, M. X. Hang et al., "Application of electronic nose on origin identification and quality grade evaluation of Jinhua and Xuanwei ham," Food and Machinery, vol. 31, pp. 114118, 2015.

[25] L. Yao, D. Y.-M. Ma, X. Z. Guo et al., "Identification and Classification of Jinhua Ham by Electronic Nose," Journal of Food Science and Biotechnology, vol. 10, pp. 1051-1056, 2012.

[26] F. Toldrá and M. Flores, "The role of muscle proteases and lipases in flavor development during the processing of drycured ham," Critical Reviews in Food Science and Nutrition, vol. 38, no. 4, pp. 331-352, 1998.

[27] J. Ruiz, J. Ventanas, R. Cava, A. Andrés, and C. García, "Volatile compounds of dry-cured Iberian ham as affected by the length of the curing process," Meat Science, vol. 52, no. 1, pp. 19-27, 1999.

[28] M. Flores, C. C. Grimm, F. Toldrá, and A. M. Spanier, "Correlations of Sensory and Volatile Compounds of Spanish "Serrano" Dry-Cured Ham as a Function of Two Processing Times," Journal of Agricultural and Food Chemistry, vol. 45, no. 6, pp. 2178-2186, 1997. 

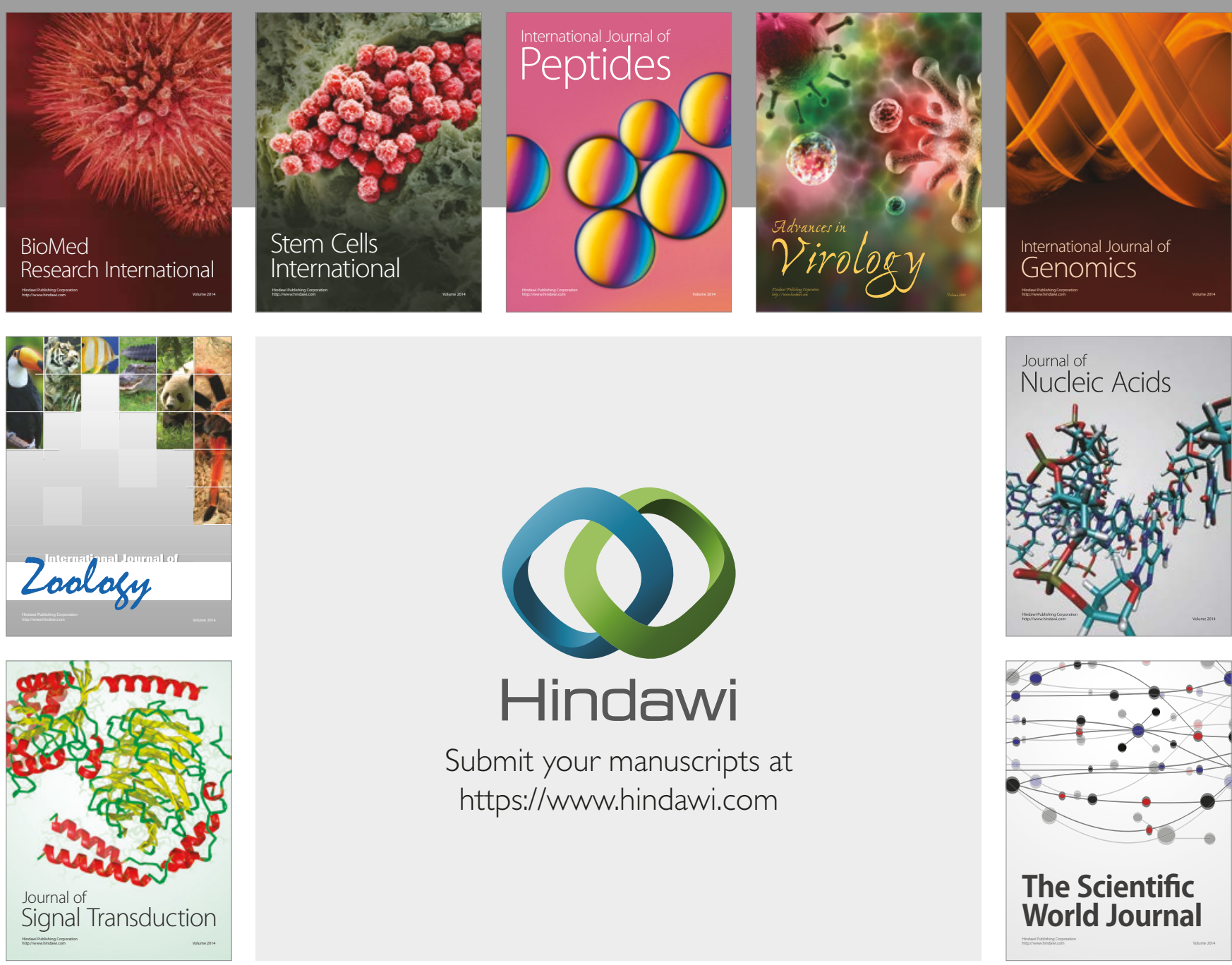

Submit your manuscripts at

https://www.hindawi.com
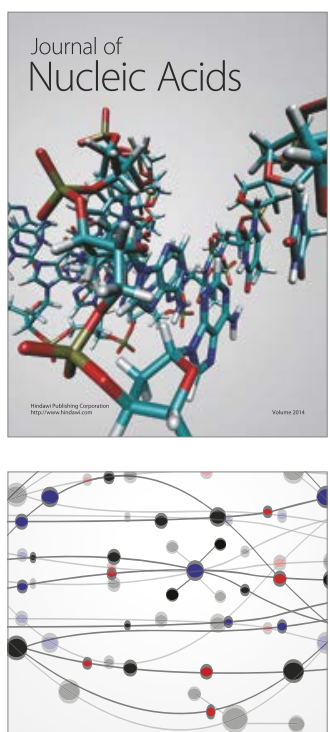

The Scientific World Journal

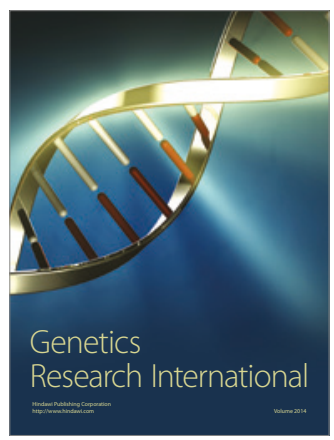

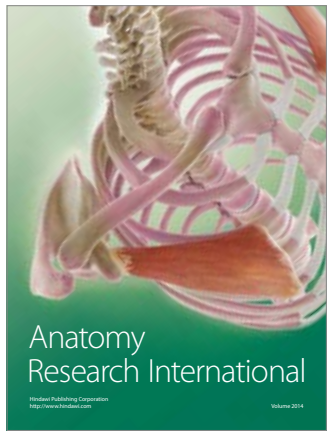

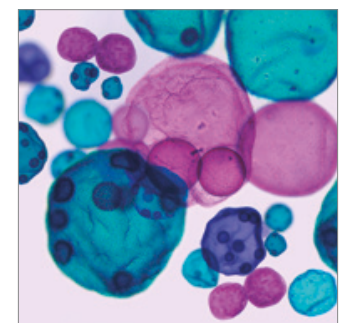

International Journal of Microbiology
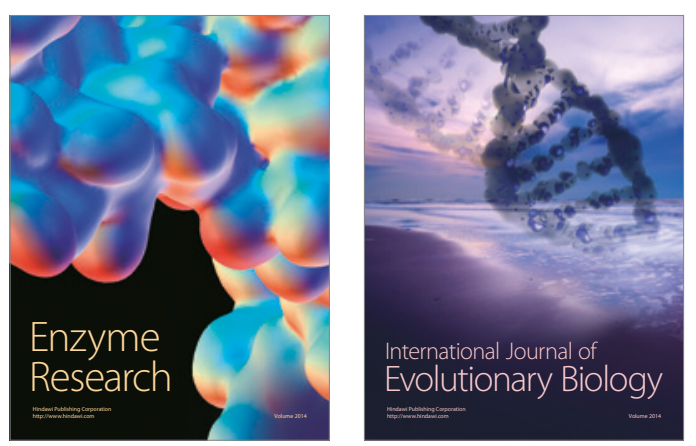
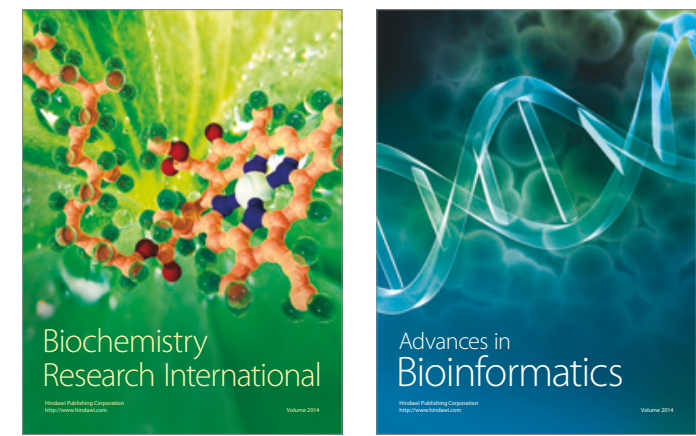

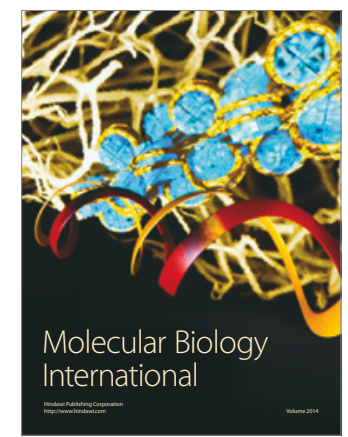

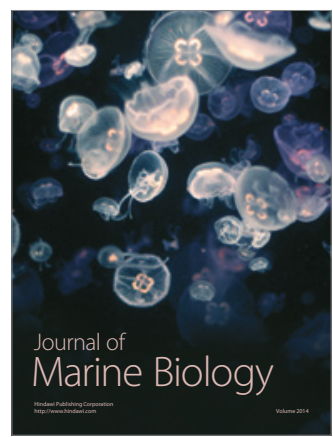

